Copyright (C) 2008 IEEE. Reprinted from

Ian B. Haya, Brent R. Petersen and Bruce G. Colpitts, "Optimum 2-D LOS MIMO performance using omni-directional antennas attained through genetic algorithms," Proceedings of the 6th Annual Communications Networks and Services Research Conference (CNSR 2008), vol. 1, (Halifax, NS, Canada), pp. 331-338, May 5-8, 2008.

This material is posted here with permission of the IEEE. Internal or personal use of this material is permitted. However, permission to reprint/republish this material for advertising or promotional purposes or for creating new collective works for resale or redistribution must be obtained from the IEEE by sending a blank email message to pubs-permissions@ieee.org.

By choosing to view this document, you agree to all provisions of the copyright laws protecting it. 


\title{
Optimum 2-D LOS MIMO Performance Using Omni-Directional Antennas Attained Through Genetic Algorithms
}

\author{
Ian B. Haya, Brent R. Petersen, and Bruce G. Colpitts \\ University of New Brunswick \\ Department of Electrical and Computer Engineering \\ Fredericton, NB, Canada - E3B 5A3 \\ ian.haya@unb.ca,b.petersen@ieee.org,colpitts@unb.ca
}

\begin{abstract}
The application of genetic algorithm (GA) optimization has been proven to be highly successful in the area of nonlinear optimizations. For a Multiple-Input, Multiple-Output (MIMO) wireless communication system, the optimum performance of the users with respect to the arrangement of antennas is a highly non-linear function which a genetic algorithm seems highly suited to. In this paper, a four-by-four MIMO system model is considered using a simplified 2-D LOS radio channel with additive white noise. Placing the users in a known arrangement, the optimum placement of the four antennas is determined through a genetic algorithm optimization using the average MSE of the four users as the fitness function. Initial results show a tendency towards an arrangement in which at least two antennas seen by the all transmitters are separated by a symbol wavelength.
\end{abstract}

\section{Introduction}

Recent work in the area of wireless communications has shown that when antenna placements in a two-by-two MIMO system are on the order of a symbol wavelength rather than the carrier wavelength, significant improvements can be made with respect to performance [1], [2]. This has given rise to the term of Signaling Wavelength Antenna Placement (SWAP) Gain to describe the advantages. The premise of this finding is that when the antennas are spaced a symbol wavelength apart, the likelihood that the channels are correlated is minimal.

Much of the MIMO work to date relies heavily on assuming a randomized multipath rich environment to realize the maximum gains from spatial diversity [3]. The fading characteristics are often modeled as Rayleigh distributions. However, in close range indoor situations, the line of sight (LOS) can often dominate the multipath components (mod- eled as Ricean distributions), minimizing the prospective gains from MIMO techniques. It is therefore necessary to examine MIMO performance in LOS situations. By strategically arranging the antennas in the system to take advantage of the SWAP Gain, an optimal placement exists that will maximize the performance of the MIMO system in a LOS situation.

However, determining the optimum placement of the antennas is seen as a highly non-linear problem that depends on the number of antennas in the system, and distribution of the users in the three-dimensional wireless communication space. It is proposed that through the use of a genetic algorithm optimization, for a given placement of users in the space, an optimum antenna placement can be achieved. Genetic algorithm optimization has seen success in many non-linear applications [4], [5], but often the results from these optimizations need interpretation. The performance of a certain antenna placement can be evaluated and the genetic algorithm then has a fitness function to base its evolutionary process on. It is possible that the algorithm can converge to a local maxima/minima point rather than reach a global solution. The presence of these vestigial structures can prove to be problem some when attempting to gain information from the results.

Initially, to investigate and simplify this process, the MIMO system has been restricted to 2-D space and only attempts to optimize the placement of the antennas through a genetic algorithm by evaluating the LOS signal.

The remainder of this paper is divided as follows. The concepts of used in this optimization are covered in Section 2. The communication system design is provided in Section 3. The GA optimization is presented in Section 4. The results of the simulation are presented in Section 5. Conclusions are drawn and discussed in Section 6. Future work is outlined in Section 7. 


\section{Overview}

\subsection{MIMO Systems}

A MIMO system makes use of multiple antennas to exploit spatial diversity. By placing the antennas some distance apart, the received signals from the same user will appear at each antenna in the system. Since the radio channel in many systems is often impaired by effects such as random noise, multi-path interference, co-channel interference (CCI), and adjacent channel interference, the resulting signals at each antenna will be different in terms of the channel impulse response [6], [7]. The noise and interference can be considered to be uncorrelated, while the message signal appearing at each antenna will retain some correlation. However, in cases where the antenna placements are similar, there exists the probability that the noise and interference will be correlated.

In general, any $M x N$ MIMO system configuration can be modeled as the matrix of channel impulse functions from the $M^{\text {th }}$ user to the $N^{\text {th }}$ antenna. Typically, a wireless communications system will rely on a large base station that handles the requests from the mobile users in the cell. An example of a mobile user placement and antenna placement configuration on a four-by-four system is shown in Fig. 1.

\subsection{Spread Spectrum Techniques}

In code division multiple access (CDMA) systems, such as the IS-95 standard and direct sequence ultra wideband (DS-UWB), multiple users are multiplexed and transmitted over the same channel by using a $K$-length pseudo random noise maximum length binary sequence, where $K$ is the spreading factor [8], [9]. The resulting signal from a single user is thus increased in bandwidth by a factor of $K$. The summation of the signals from the total users produces an orthogonal signal set such that the original users signal can be de-multiplexed from the resultant signal by using the same generating code on the receive end of the channel.

Some of the disadvantages of CDMA schemes are that they are affected more by multiple access interference (MAI) and intersymbol interference (ISI) [10]. To allow for this, a spreading factor greater than the expected capacity is used, resulting in a greater grade of service (GOS) at the expense of more bandwidth.

\subsection{Symbol Wavelength}

The symbol wavelength, $\lambda_{T}$, is defined as

$$
\lambda_{T}=\frac{c}{f_{g}}
$$
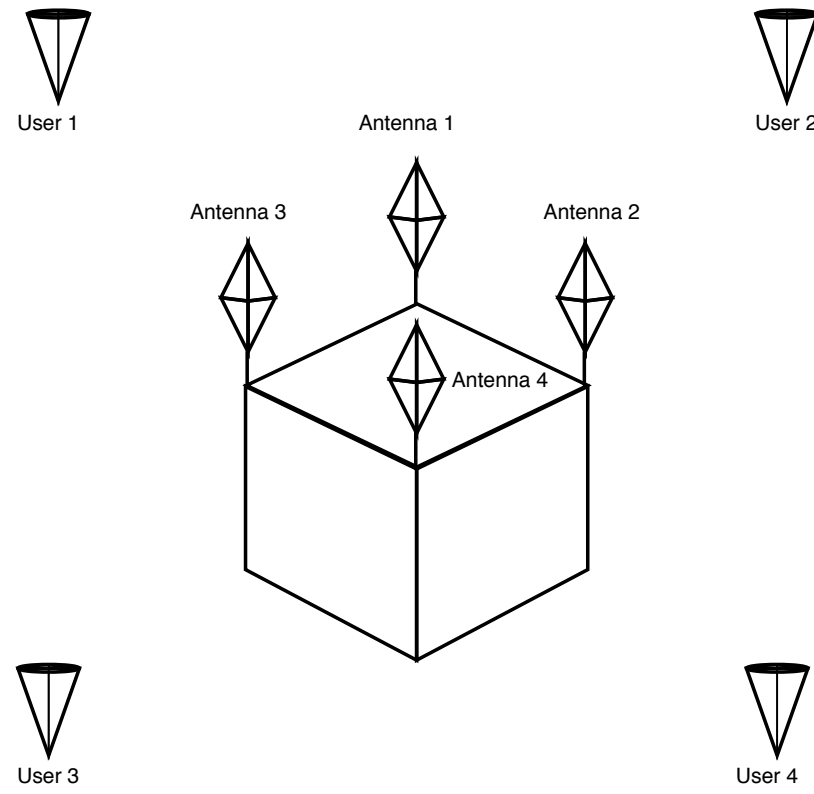

Figure 1. A depiction of a four-by-four arrangement for a multiple-input, multipleoutput (MIMO) system with mobile users placed around the antenna arrangement at the center of the cell.

where $c$ is the speed of light and $f_{g}$ is the symbol rate. It has been shown in [1], [11] that by placing antennas on the order of $\lambda_{T}$ that a greater diversity gain is achieved as opposed to traditional carrier wavelength spacing. For purposes of comparison, the antenna separations in the genetic algorithm optimization simulation have been normalized with respect to the symbol wavelength.

\subsection{Radio Channel}

The mobile radio channel is inherently noisy and cluttered with interference from other mobiles and multi-path reflections. The overall performance of wireless communication system is concerned with the multiple ways to reduce the Signal to Interference plus Noise Ratio (SINR). In 1948, Shannon demonstrated that through proper encoding, errors can be reduced to any desired level without sacrificing the rate of information transfer [12]. This led to the what is know as Shannons channel capacity formula given by

$$
C=B \log _{2}\left(1+\frac{S}{N}\right),
$$

where $C$ is the channel capacity (bits per second), $B$ is the transmission bandwidth ( $\mathrm{Hz}), S$ is the signal power $(\mathrm{W})$, and $N$ is the noise power (W). 


\subsection{LMS Adaptive Filter}

The least mean square (LMS) adaptive filter is another proven concept that has shown great performance and widespread use due to its ease of implementation [13], [14]. The basic setup of a LMS adaptive filter is shown in Fig. 2.

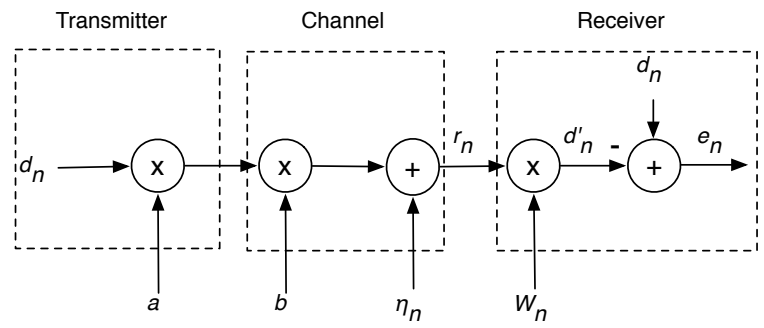

\section{Figure 2. A block diagram of the described simple LMS adaptive filter.}

In this arrangement, the data stream to be transmitted is given by $d_{n}, a$ denotes the spreading code applied to the data, $b$ represents the wireless channel response, $\eta_{n}$ is the AWGN, $r_{n}$ is the signal received at the antenna, $W_{n}$ is the adaptive filter coefficient, $d_{n}^{\prime}$ is the filtered received signal, and $e_{n}$ is the error associated with the filtered received signal.

During training, the receiver knows dn, as the training sequence would be programmed into the adaptive filter logic. It will then update the filter coefficient $W_{n}$ according to

$$
W_{n+1}=W_{n}+\mu e_{n} r_{n},
$$

where $W_{n+1}$ is the updated filter coefficient, $W_{n}$ is the current filter coefficient, and $\mu$ is the LMS adaptation constant, which is chosen to be small enough such that the filter will converge. If $\mu$ is chosen to be too large, the adaptation will diverge and the minimum mean square error (MMSE) will not be reached.

After the filter has finished processing the training sequence, the filter then switches from operating on the training sequence and continues to adapt from the incoming signal. Ideally at this point the adaptive filter has converged and has successfully performed the channel inversion to create a matched filter and remains at the global minimum rather than diverging off to some other local minimum.

\subsection{Genetic Algorithms}

Genetic algorithm (GA) optimization borrows on the ideas of evolution found in the everyday biology of living organisms. First discussed in Charles Darwins Origin of Species, the concept is that every living organism that exists today is a result of a process of evolution over the many generations that the population has existed for over great lengths of time. Within every cell of an organism, a genetic blueprint is contained within a chemical substance called deoxyribonucleic acid (DNA). This chemical substance is in a double helical structure and contains continuous base pairs of the nucleotides adenine (A), thymine (T), guanine $(\mathrm{T})$ and cytosine $(\mathrm{C})$. The sequencing of these nucleotides provides the basic genetic code that is capable of completely reproducing the organism in which the DNA is contained [4], [5].

Translating this to science and engineering problems, a set of possible solutions becomes the population of living organisms. This population is then evaluated to determine their fitness to performing the desired goal defined in the problem. Such as in nature, the individuals are then subjected to a survival of the fittest evaluation, where only a portion of the top performing individuals are retained for the next generation. These top performing individuals are also chosen to be the parents for the succeeding population. These parents then generate offspring to fill the population. The offspring are generated in primarily two mechanisms, through crossover and mutation.

One of the advantages of genetic algorithms is that they are capable of operating on a problem that has a very large set of possible solutions [4]. A problem with a large set of solutions may not be computationally practical to investigate through brute forcing methods. This leads to the advantage that genetic algorithms will often lead to solutions that would otherwise not have been reached through common numerical techniques.

\section{Communication System Design}

\subsection{MIMO Setup}

For the genetic algorithm optimization simulation, a four-by-four MIMO system arrangement was chosen as a model. This model was configuration was chosen since it would be complex enough to exhibit characteristics of the nonlinearities of the problem without being overly computationally complex. In terms of the channel impulse functions between the users and the base stations, the channel impulse function matrix is given by

$$
\mathbf{h}(t)=\left(\begin{array}{llll}
h_{11}(t) & h_{12}(t) & h_{13}(t) & h_{14}(t) \\
h_{21}(t) & h_{22}(t) & h_{23}(t) & h_{24}(t) \\
h_{31}(t) & h_{32}(t) & h_{33}(t) & h_{34}(t) \\
h_{41}(t) & h_{42}(t) & h_{43}(t) & h_{44}(t)
\end{array}\right)
$$


which has the corresponding Fourier transform

$$
\mathbf{H}(f)=\left(\begin{array}{cccc}
H_{11}(f) & H_{12}(f) & H_{13}(f) & H_{14}(f) \\
H_{21}(f) & H_{22}(f) & H_{23}(f) & H_{24}(f) \\
H_{31}(f) & H_{32}(f) & H_{33}(f) & H_{34}(f) \\
H_{41}(f) & H_{42}(f) & H_{43}(f) & H_{44}(f)
\end{array}\right) .
$$

\subsection{Signal Generation}

For the purpose of the genetic algorithm optimization, a spreading factor of $K=8$ was chosen. This was chosen as a compromise between giving the coded signals enough of a spread to be recovered after noise was added to the channel, and the computational complexity associated with increasing the bandwidth of the transmitted signals. The spread spectrum spreading codes were generated randomly with complex values and unit energy.

\subsection{Radio Channel Modelling}

In the described genetic algorithm optimization, the radio channel was modeled as being a pure line of sight (LOS) radio channel. In a pure LOS radio channel, the aspects of multi-path interference and ground effects are ignored. The attenuation of the signal is inversely proportional to the square of the distance. This gives rise to a path loss exponent, $n$, of 2 , and determines the received power by

$$
P_{r}(d)=P_{r}\left(d_{0}\right)\left(\frac{d_{0}}{d}\right)^{n},
$$

where $P_{r}$ is the received power $(\mathrm{W}), d_{0}$ is a reference distance close to the base station $(\mathrm{m})$, and $d$ is the distance from the base station (m). Also, for the purpose of this simulation, the antennas were modelled as omni-directional antennas such that the isotropic gain was unity.

The next point to consider is the propagation of the signals is considered to be in free space and is therefore taken as $c$, the speed of light. This gives rise to a time delay for the propagation from the mobile to the antennas. Using the two points of path loss and time delay, the entries of (2) can now be expressed as a function of the distance from mobile to the antennas to give

$$
H_{i j}(f)=M_{i j} \mathrm{e}^{j 2 \pi f_{g} t_{i j}},
$$

where $M_{i j}$ is the resulting attenuation of the signal from the $i^{\text {th }}$ mobile to the $j^{\text {th }}$ antenna, $t_{i j}$ is the time delay associated with the signal from the $i^{\text {th }}$ mobile to the $j^{\text {th }}$ antenna, and $f_{g}$ is the symbol rate.

The sources of interference that arise in this simulation are MAI and additive white Gaussian noise (AWGN). Complex random noise was generated and added to the received signals at each antenna. The noise variance, $\sigma_{n}$ was chosen to give a signal to noise ratio of $40 \mathrm{~dB}$ at each antenna.

\subsection{Signal Extraction}

The LMS adaptive filter was applied to each received signal at each antenna to extract the original data stream. The LMS adaptation constant, $\mu$, was set to $2^{-5}=0.03125$. For the purpose of this simulation, the entire length of the data stream was considered known, and the adaptive filter was allowed to train on the whole data sequence. The length of the data sequence was set to be a total of 1024 bits. The adaptive filter was assumed to have converged to the global minimum and the MSE was then calculated over the second half of the data stream (512 bits). The value for the MSE over the second half of the data stream was taken as the MMSE value for that user.

\section{GA Optimization Design}

\subsection{Antenna DNA}

In this simulation, the placement of the four antennas is chosen as the individuals DNA structure. The antenna placement is evaluated only in two dimensions, so antenna placement contains an $x$ and $y$ co-ordinate describing its placement within the cell. Since each individual is made up of four antenna placements, the individuals of the population can be described by

$$
\mathbf{D N A} \mathbf{A}_{\mathbf{i}}=\left[\begin{array}{llllllll}
x_{1} & y_{1} & x_{2} & y_{2} & x_{3} & y_{3} & x_{4} & y_{4}
\end{array}\right] .
$$

This could be modified to account for $N$ antennas by simply extending (8) by adding $x$ and $y$ co-ordinates for each additional antenna. Each element is referred to as an allele of the individual, which in traditional genetics is a sequence of DNA code that is responsible for a particular characteristic in an individual. A constraint was placed on the DNA of the antennas to limit the total distance the antennas were placed from the origin. This was imposed to simulate some cost function associated with a given antenna placement structure.

\subsection{Fitness}

For each generation of individuals that was created, it was necessary to evaluate the performance of the individuals according to a fitness function, how well the individuals were capable of achieving the specified goal. In a wireless communications system, the goal is ultimately to deliver the information reliably and efficiently. The two most common metrics that measure a systems performance in a wireless communications channel are bit error rate (BER) and the MMSE described in section VI [15]. For each individual of the antenna placement population, four MMSE values were determined, one for each user in the population. To obtain a 
single score for each individual in the population, the fitness function, $\phi$, was given by

$$
\phi=\frac{1}{\frac{1}{N} \sum_{i=0}^{N} \mathrm{MMSE}_{i}},
$$

where $\mathrm{N}$ is the number of users.

Upon calculating $\phi$, the population can then be ranked according to the resulting scores. Since a small MMSE is desired, the best scoring individuals will have a large value for $\phi$.

\subsection{Generating Populations}

In order to evolve, the next generation of individuals needs to inherit the properties of the top performing individuals from the previous generation and attempt to improve upon them. The portion of top performers retained for the succeeding generation was set at $10 \%$. These top performers were chosen as the parents to generate the next population through the techniques of crossover and mutation.

\subsubsection{Crossover}

To generate a new individual based on the genetic technique of crossover, two parents are randomly chosen from the top performing population. A binary crossover vector is randomly generated having equal length of the DNA code. The new individual is created by using a combination of the alleles found on in the DNA codes of the two parents. In this case, on the loci (location of allele, or DNA code index) where the crossover vector is a 0 , the offspring will inherit the attribute found at the same site as Parent 1.

\subsubsection{Mutation}

The second method by which new individuals are created is through the process of mutation. This method involves adding random perturbations to the genetic code to create new individuals that result from a morphing of the parent. In nature, this process is invoked to increase the available genetic content in a population. The mathematical equivalent to this is to give the population the ability to evolve towards a global optimization rather than remain at some local minima. Often, it is quite possible as well for individuals to be created with similar performance, but vastly different characteristics.

To generate a new individual via mutation, first, an individual is randomly selected from the top performer population to be mutated. A mutation vector of the same length as the DNA code is then generated by randomly selecting a perturbation from a zero mean normal distribution. This perturbation vector is then added to the selected parent to

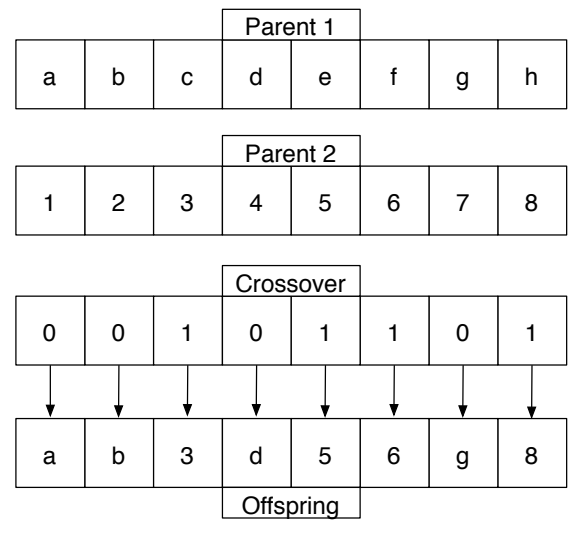

Figure 3. A depiction of the crossover process in which a new offspring is created by inheriting attributes from two selected elite parents.

create a new individual that is a resultant of the morphed values.

\begin{tabular}{|l|l|l|l|l|l|l|l|}
\cline { 3 - 6 } \multicolumn{1}{l|}{} & \multicolumn{3}{|c|}{ Parent } & \multicolumn{3}{l|}{} \\
\hline 1 & 2 & 3 & 4 & 5 & 6 & 7 & 8 \\
\hline
\end{tabular}

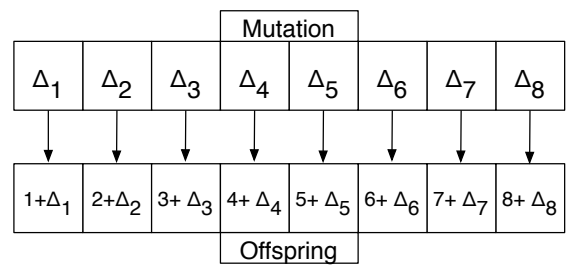

Figure 4. A depiction of the mutation process in which a new offspring is created by adding perturbations to the attributes of a randomly selected elite individual.

The standard deviation of the mutation vector, $\sigma_{m}$, was given a starting value, $\sigma_{m_{0}}$, and chosen as $0.1 \lambda_{T}$. Another characteristic of population genetics is that often when a population is young, it is necessary for the mutations to be large and abundant. As the population evolves, it becomes more specialized and large mutations often appear to provide no further advantages. Also, the value of $\sigma_{m}$ will determine the variance associated with a population. In order to meet some predefined convergence criteria, it is then necessary for the $\sigma_{m}$ to decrease as the population becomes more specialized. This gives rise to a degradation factor, $\alpha$, to determine the value of $\sigma_{m}$ for the next population. The 
calculation of the $\sigma_{m}$ is therefore give by

$$
\sigma_{m}(\gamma)=\sigma_{m_{0}} \alpha^{\gamma-1}
$$

where $\gamma$, is the generation index. A value for $\alpha$ was chosen as 0.97 .

\section{Simulation Results}

The simulation was coded as a MATLAB ${ }^{\circledR}$ script file. Several different user orientations were considered and the output of the optimizations was retained for each generation. For each user orientation, the population size was set to 100 individuals. The number of generations that were simulated was also 100 . The selection criterion was retained as the top $10 \%$ performing individuals. A crossover ratio of 0.5 was chosen. This meant that $50 \%$ of the new individuals that were created were done so by using the crossover technique, while the remaining $50 \%$ were generated through mutation.

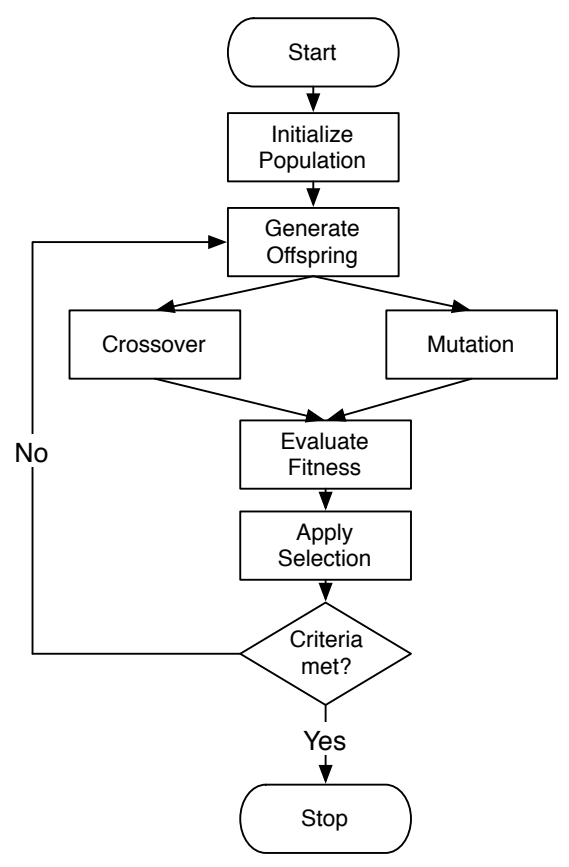

\section{Figure 5. A generalized flow chart for the ge- netic algorithm optimization process.}

A second run of the simulation was repeated for the same user configurations, but this time choosing a crossover ratio of 0 . This meant that the generation of new individuals was done through pure mutation.

Fig. 6 shows an example of one of the mobile user placements for which the simulation was run. This particu- lar configuration shows the mobile users equally separated around the origin of the cell, each at a distance of $\lambda_{T}$.

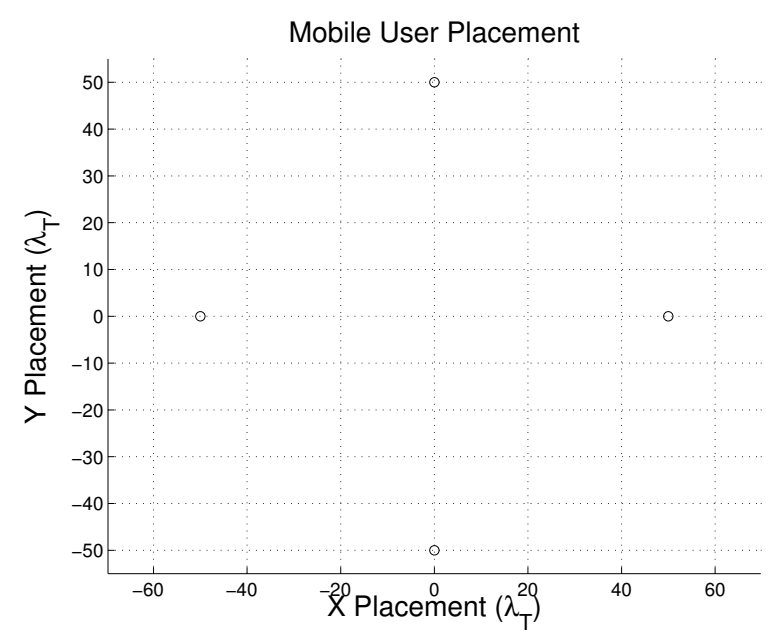

\section{Figure 6. A configuration used for the place- ment of the mobile users in the cell.}

For the initial simulation run, using a crossover ratio of 0.5 , the genetic algorithm tended towards an arrangement in which at least two antennas are separated by $\lambda_{T}$ as seen by the mobile users and asymmetry (Fig. 7).

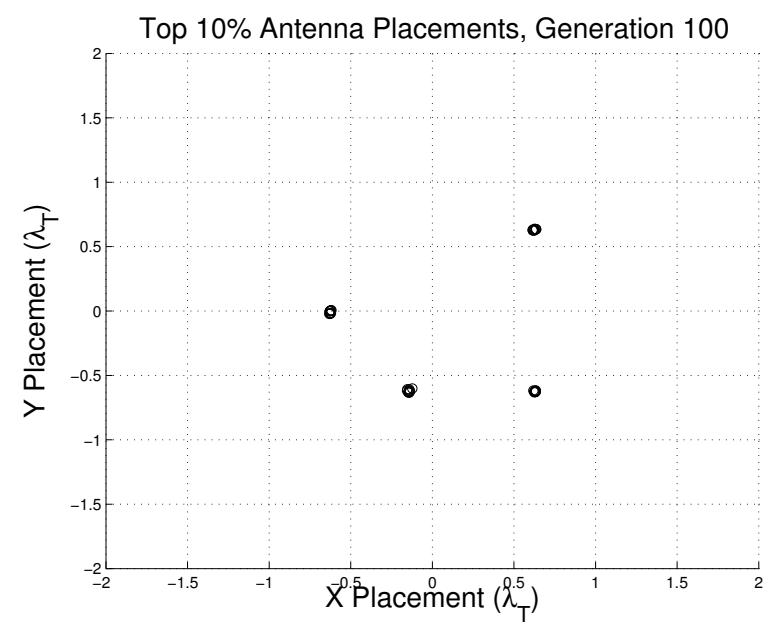

Figure 7. Antenna placements for the top $10 \%$ using the mobile user placement in Fig. 6 and a crossover ratio of 0.5 after $\mathbf{1 0 0}$ generations.

For the next simulation run, using a crossover ratio of 0 , e.g. pure mutation, the genetic algorithm tended towards 
a different arrangement (Fig. 8). This arrangement also shows asymmetric qualities as well as having at least two antennas separated by $\lambda_{T}$ as seen by the mobile users. In fact, this arrangement is a 180 degree rotation of the same antenna placement achieved through crossover, which can be considered the same result given the symmetry in the original mobile user placement. This shows that either through pure mutation, or including some degree of crossover, the same results can be achieved.

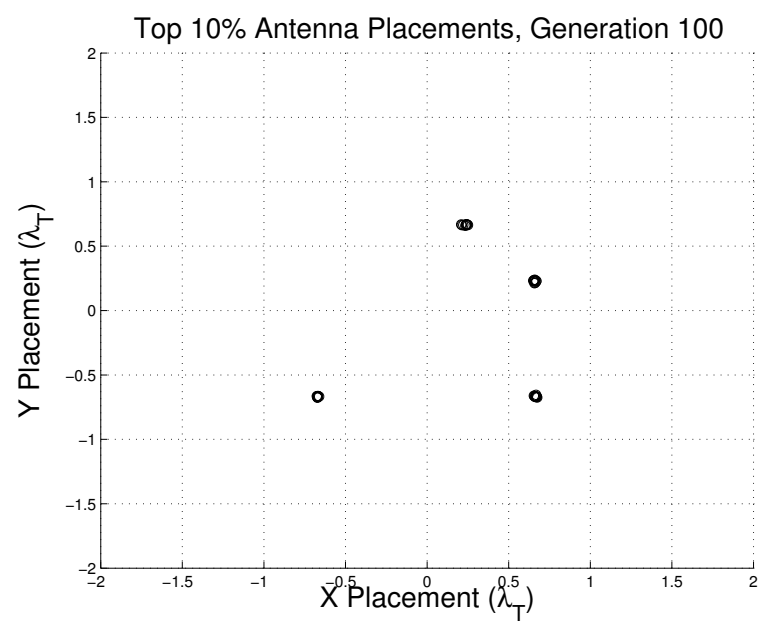

Figure 8. Antenna placements for the top 10\% using the mobile user placement in Fig. 6 using a crossover ratio of 0 after 100 generations.

\section{Conclusion}

In this paper, a method for determining the optimum MIMO performance using omni-directional antennas in an array over LOS radio channels through genetic algorithm optimization was presented. The initial results showed a tendency for the positions of the antenna placements to be in an asymmetric arrangement in which at least two antennas are separated by $\lambda_{T}$ as seen by the mobile users. The concept of asymmetric placements can be accepted in the notion that it maximizes diversity, since symmetric configurations will produce received signals with no relative phase delay, and thus increasing the difficulty of separating the signals.

One of the reinforcing concepts that was found was that in all instances, the antenna arrangements produced a configuration in which at least two antennas were separated on the order of $\lambda_{T}$. This agrees with the previous findings that SWAP Gain can be achieved when antenna place- ments use this separation. It should be also noted that the arrangements were not likely to increase the antenna separation much more than $\lambda_{T}$, indicating that the performance loss from the degradation in signal power due to the excess power lost affects the placement of the antennas.

\section{Future Work}

In the future, the scope of this work will be extended in many ways. The design space for the antenna placement and user placement will be extended to three-dimensional space. Additionally, the performance of the system could also be evaluated for increasing numbers of users, and the performance degradation associated with the increased demand. Since this investigation was only evaluated for certain known placements of the users in the cell, this work could also be extended to consider more random placements of the users to gain an insight into a configuration that would provide optimum performance for all placements of users. Adding more complexity to the antenna model by adding radiation patterns rather than considering the simple omnidirectional case could also prove useful for real world implementation.

The main focus of the future work however, will be incorporating reflector elements as part of the design to purposely introduce random reflections to create additional multipath components that will be received by the antennas. By adding these reflectors to the system, it will enable the MIMO system to behave as a mutlipath rich environment in what was previously dominated by the LOS component. The number, placement, size, shape, and orientation of these reflectors will become a part of the genetic algorithm.

\section{Acknowledgment}

This research was funded by a Post-graduate Scholarship from the Natural Sciences and Engineering Research Council, by the Atlantic Innovation Fund from the Atlantic Canada Opportunities Agency, and by Bell Aliant, our industrial partner.

\section{References}

[1] G. Zhu, B. R. Petersen, and B. G. Colpitts, "Signalling wavelength in an antenna array for space-time wireless over LOS channels," in Proceedings of the 3rd Annual Communications Networks Services and Research Conference (CNSR 2005), vol. 1, Halifax, NS, Canada, May 2005, pp. 69-73.

[2] V. V. N. S. Polu, B. G. Colpitts, and B. R. Petersen, "Symbol-wavelength MMSE gain in a multi-antenna 
UWB system," in Proceedings of the 4th Annual Communications Networks and Services Research Conference (CNSR 2006), vol. 1, Moncton, NB, Canada, May 2006, pp. 95-99.

[3] J. Yang and S. Roy, "On joint transmitter and receiver optimization for multiple-input multiple-output (MIMO) transmission systems," IEEE Trans. Commun., vol. 42, no. 12, pp. 3221-3231, Dec 1994.

[4] D. Fogel, "Evolutionary computing," IEEE Spectr., vol. 37, no. 2, pp. 26-32, Feb 2000.

[5] D. Anastassiou, "Genomic signal processing," IEEE Signal Process. Mag., vol. 18, no. 4, pp. 8-20, Jul 2001.

[6] B. Widrow, P. Mantey, L. Griffiths, and B. Goode, "Adaptive antenna systems," Proc. IEEE, vol. 55, no. 12, pp. 2143-2159, Dec 1967.

[7] J. H. Winters, "Optimum combining in digital mobile radio with cochannel interference," IEEE J. Sel. Areas Commun., vol. 2, no. 4, pp. 528-539, Jul 1984.

[8] A. J. Viterbi, CDMA: Principles of Spread Spectrum Communication. Reading, MA, USA: AddisonWesley Publishing Co. Inc., 1995.

[9] A. Soong and W. Krzymien, "Effect of antenna diversity on performance of reference symbol assisted interference cancellation in CDMA wireless systems," in Conf. Rec. 1997 IEEE 6th International Conference on Universal Personal Communications (ICUPC), vol. 1, Oct 1997, pp. 202-207.

[10] P. Jung and P. Alexander, "A unified approach to multiuser detectors for CDMA and their geometrical interpretations," IEEE J. Sel. Areas Commun., vol. 14, no. 8, pp. 1595-1601, Oct 1996.

[11] H. Yanikomeroglu and E. S. Sousa, "Antenna gain against interference in CDMA macrodiversity systems," IEEE Trans. Commun., vol. 50, no. 8, pp. 1356-1371, Aug 2002.

[12] T. S. Rappaport, Wireless Communications. Upper Saddle River, New Jersey, USA: Prentice Hall PTR, 2001.

[13] R. Ziegler and J. Cioffi, "A comparison of least squares and gradient equalization for multipath fading in wideband digital mobile radio," in Conf. Rec. IEEE Globecom 89, vol. 1, Nov 1989, pp. 102-105.

[14] G. Cherubini, E. Eleftheriou, and S. Ölçer, "Filtered multitone modulation for very high-speed digital subscriber lines," IEEE J. Sel. Areas Commun., vol. 20, no. 5, pp. 1016-1028, Jun 2002.
[15] J. H. Winters, "Upper bounds for the bit error rate of optimum combining in digital mobile radio," in Conf. Rec. IEEE Globecom 84, vol. 1, Nov 1984, pp. 173178. 